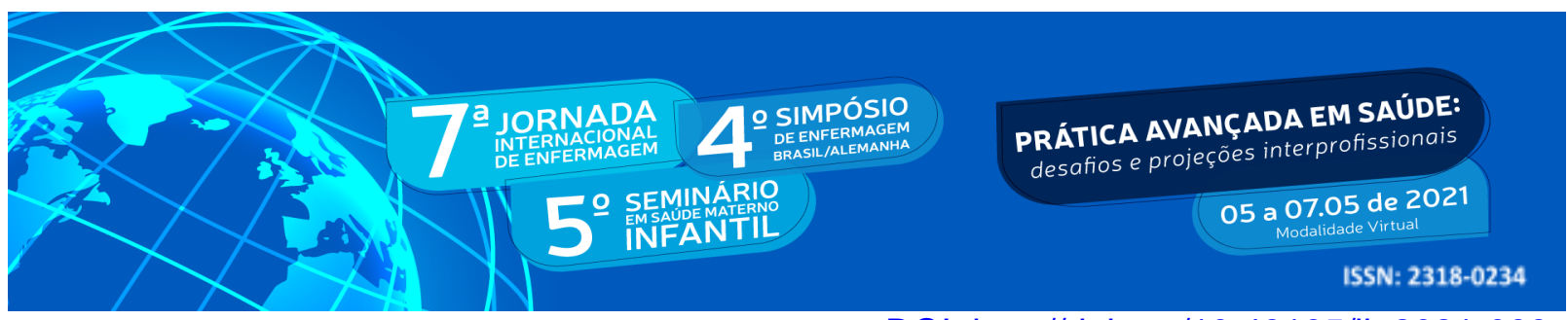

DOI: http://doi.org/10.48195/jie2021-099

\title{
EDUCAÇÃO EM SAÚDE NA PROMOÇÃO DA AMAMENTAÇÃO NA ATENÇÃO PRIMÁRIA À SAÚDE: UMA REVISÃO INTEGRATIVA ${ }^{1}$
}

\author{
Sidney Bruno Lima da Silva ${ }^{2}$; Ketyllem Tayanne da Silva Costa ${ }^{3}$; Ana Luiza Santos \\ Quirino $^{4}$; Maria de Lourdes Alves da Cruz ${ }^{5}$; Larissa Beatriz Francisca de Souza ${ }^{6}$; Fábia \\ Barbosa de Andrade ${ }^{7}$
}

\section{RESUMO}

Objetivou-se analisar o impacto da educação em saúde na promoção da amamentação na Atenção Primária à Saúde, a partir de estudos disponíveis na literatura. Para tanto, foi realizada uma revisão integrativa da literatura, norteada pela questão: "Como a educação em saúde, na atenção primária à saúde, impacta na promoção da amamentação?". Buscou-se publicações nas fontes de dados: Medical Literature Analysis and Retrieval System Online, Biblioteca Virtual em Saúde, Cumulative Index to Nursing and Allied Health Literature, Scopus Content Overview e Embase. Obteve-se 332 artigos, selecionando-se 7 para a amostra. Os resultados foram positivamente favoráveis quanto aos efeitos que as ações de intervenção em saúde possuíam sobre a promoção, proteção e apoio ao aleitamento materno no âmbito da atenção primária. Conclui-se que as intervenções educativas promovidas são ferramentas eficazes para a prática da amamentação, permitindo a inclusão das mães e sua rede de apoio no processo de aprendizagem.

Palavras-chave: Aleitamento Materno; Atenção Primária à Saúde; Educação em Saúde.

\section{ABSTRACT}

The objective was to analyze the impact of health education on the promotion of breastfeeding in Primary Health Care, based on studies available in the literature. For that, an integrative literature review was conducted, guided by the question: "How does health education in primary health care impact on the promotion of breastfeeding?". We searched for publications in the data sources: Medical Literature Analysis and Retrieval System Online, Virtual Health Library, Cumulative Index to Nursing and Allied Health Literature, Scopus Content Overview and Embase. 332 articles were obtained, with 7 selected for the sample. The results were positively favorable regarding the effects that health intervention actions had on the promotion, protection and support of breastfeeding in the context of primary care. It is concluded that the educational interventions promoted are effective tools for the practice of breastfeeding, allowing the inclusion of mothers and their support network in the learning process.

Key Words: Breast Feeding; Health Education; Primary Health Care.

\footnotetext{
${ }^{1}$ Revisão integrativa da literatura.

${ }^{2}$ Estudante do Curso de Enfermagem. UFRN. E-mail: sidney.natal10@gmail.com

${ }^{3}$ Estudante do Curso de Enfermagem. UFRN. E-mail: ketyllemcosta@gmail.com

${ }^{4}$ Estudante do Curso de Enfermagem. UFRN. E-mail: luizasantos699@gmail.com

${ }^{5}$ Estudante do Curso de Enfermagem. UFRN. E-mail: dudinhalves.29@gmail.com

${ }^{6}$ Estudante do Curso de Enfermagem. UFRN. E-mail: larissa.beatriz@ufrn.edu.br

${ }^{7}$ Orientadora. Doutora em Ciências da Saúde. UFRN. E-mail: fabiabarbosabr@gmail.com
} 


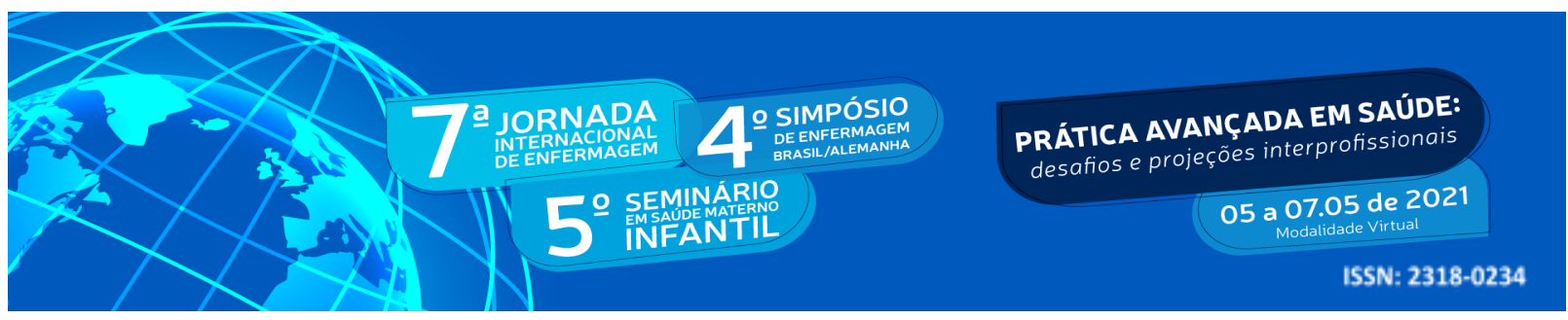

\section{INTRODUÇÃO}

A infância configura-se como um período em que ocorre o desenvolvimento de grande parte das potencialidades humanas (BRASIL, 2015). Nesse sentido, o aleitamento materno (AM) caracteriza-se como meio cientificamente eficaz no crescimento saudável e na redução da morbimortalidade infantil, em decorrência do seu valor nutricional; sua proteção imunológica; menor contaminação; redução do risco de obesidade e diabetes; declínio de infecções respiratórias e episódios diarreicos (VICTÓRIA et al., 2016).

A Organização Mundial da Saúde (OMS), estabelece três importantes classificações para o aleitamento materno, sendo: o aleitamento materno exclusivo (AME), em que deve ser ofertado apenas o leite materno ao bebê até os 6 meses de vida; o aleitamento materno predominante, que incorpora à dieta da criança água e sucos de frutas; e o aleitamento materno complementado, quando são adicionados à alimentação da criança quaisquer alimentos sólidos ou líquidos, inclusive fórmulas infantis e leite não humano (OMS, 2007).

No tocante à assistência em saúde, a Atenção Primária à Saúde (APS), caracteriza-se como a principal e mais adequada forma de acesso das pessoas aos serviços de saúde (TASCA et al., 2020). Portanto, constitui-se como um ambiente incentivador na manutenção da prática de amamentação, tanto durante o pré-natal, quanto no período puerperal, oportunizando a identificação de fatores influentes no desmame precoce e estabelecendo medidas de intervenção (MELO et al., 2019).

Nesse sentido, o Ministério da Saúde adotou, em 2008, a Rede Amamenta Brasil, apoiada nos princípios da educação crítico-reflexiva, voltada para a revisão e o matriciamento dos processos de trabalho interdisciplinar na APS (BRASIL, 2009). Posteriormente, pela Portaria ${ }^{\circ} 1.920$, de 5 de setembro de 2013, tal política foi incorporada à Estratégia Nacional de Promoção da Alimentação Complementar Saudável (Enpacs), resultando na Estratégia Amamenta e Alimenta Brasil. Esta objetivou a qualificação das ações de promoção, proteção e apoio ao aleitamento materno e a alimentação complementar saudável para crianças menores de 2 anos de idade (BRASIL, 2013).

Nesse ínterim, é imprescindível a promoção da educação em saúde, considerando as vivências e conhecimentos das pessoas envolvidas, com ações participativas para construção do pensamento crítico-reflexivo (VIEIRA; MATIAS; QUEIROZ, 2021). Portanto, o presente 


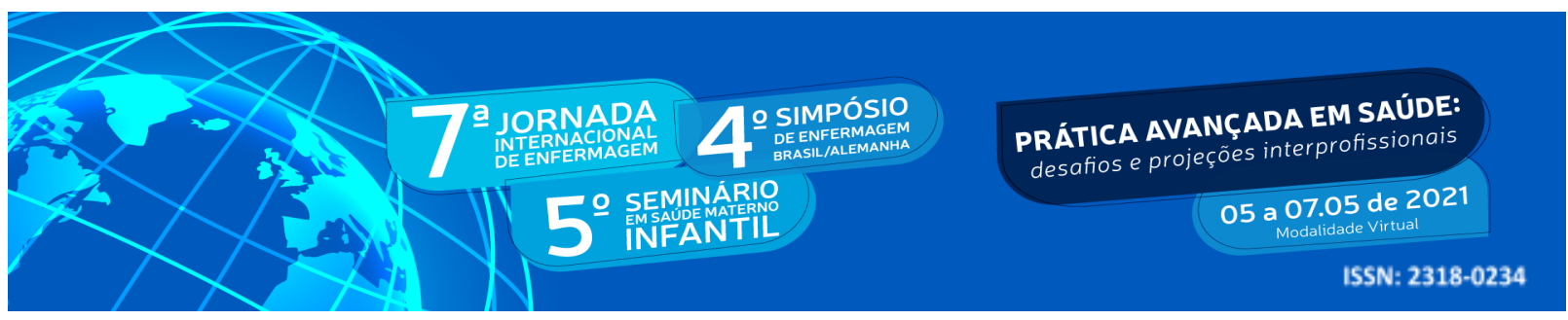

estudo justifica-se por evidenciar a relação existente entre as práticas de educação em saúde e a promoção do aleitamento materno no âmbito da Atenção Primária à Saúde, a partir do pressuposto da importância da amamentação para o desenvolvimento saudável infantil.

\section{OBJETIVO}

Analisar o impacto da educação em saúde na promoção da amamentação no contexto da Atenção Primária à Saúde, a partir de estudos disponíveis na literatura.

\section{METODOLOGIA}

Trata-se de uma revisão integrativa da literatura, realizada no mês de fevereiro do ano de 2021. Para o direcionamento do estudo, foi formulada a seguinte questão norteadora: “Como a educação em saúde, na atenção primária à saúde, impacta na promoção da amamentação?”. Para a construção desta, foi utilizado o acrônimo PICO (População/Paciente, Intervenção, Controle e Outcome/Desfecho), com as seguintes definições: P - gestantes, puérperas e profissionais da saúde; I - educação em saúde; O - promoção do aleitamento materno. $\mathrm{O}$ elemento mnemônico $\mathrm{C}$ (Controle) não foi incorporado à questão.

Realizou-se, então, a busca dos descritores no vocabulário controlado Descritores em Ciências da Saúde (DeCS) e foram determinados "Educação em Saúde", "Aleitamento Materno", e "Atenção Primária à Saúde"; além de equivalentes no idioma inglês confirmados conforme o Medical Subject Headings (MeSH), sendo: "Health Education", "Breast Feeding" e "Primary Health Care". Realizou-se o cruzamento dos termos indexados pelo operador booleano "AND".

As fontes de dados consultadas foram, a saber: Medical Literature Analysis and Retrieval System Online (Medline), Biblioteca Virtual em Saúde (BVS), Cumulative Index to Nursing and Allied Health Literature (CINAHL), Scopus Content Overview (SCOPUS) e Embase. Ainda, a seleção inicial dos estudos foi pautada em alguns critérios de elegibilidade.

Como critérios de inclusão, adotou-se: estudos primários publicados entre os anos de 2016 e 2021, nos idiomas português, inglês e espanhol e que atendam ao objetivo da revisão. Já os critérios de exclusão foram: estudos publicados fora do período determinado, fora dos idiomas selecionados, publicações em formato de editorial, carta ao editor, dissertação, tese, revisão da literatura e documentos duplicados e com resultados inconclusivos. 


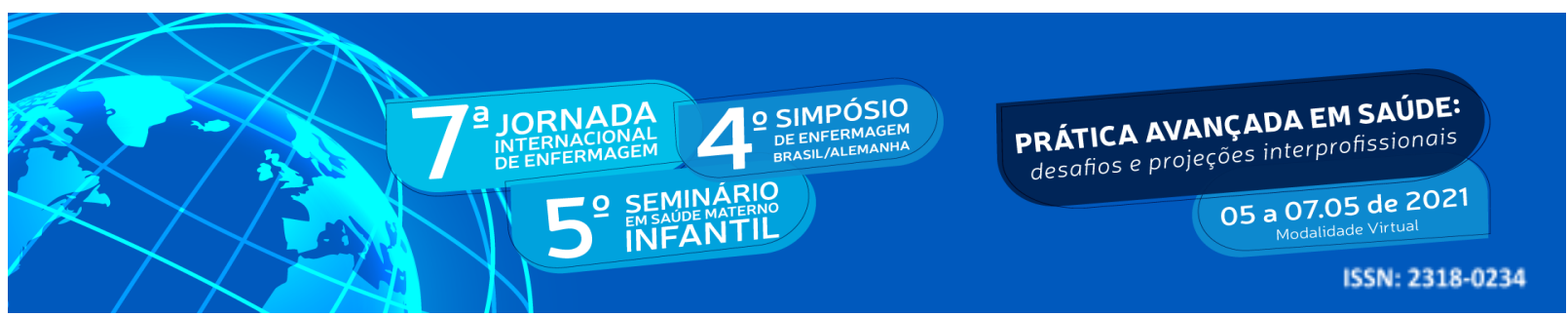

Nesta perspectiva, a busca foi realizada, simultaneamente, por três pesquisadores, de forma independente. Para isso, utilizou-se como suporte o software State of the Art through Systematic Review (StArt), um sistema computacional gratuito, que possibilita inserir, em sua interface, o protocolo de pesquisa e os resultados das buscas nas bases de dados, a fim de organizá-los conforme a seleção dos estudos.

Inicialmente foram encontradas 332 publicações, seguindo os critérios de elegibilidade estabelecidos, dos quais foram retirados 42 por estarem duplicados. Após, foi feita uma triagem inicial, a partir da leitura dos títulos e resumos, selecionando-se 25 estudos. Em uma segunda triagem, foi realizada uma leitura criteriosa dos artigos completos, em que pôde-se chegar à amostra final de 7 artigos para compor a presente revisão. Por fim, o processo de triagem foi representado por meio de um fluxograma conforme a figura 1.

Figura 1 - Triagem dos estudos

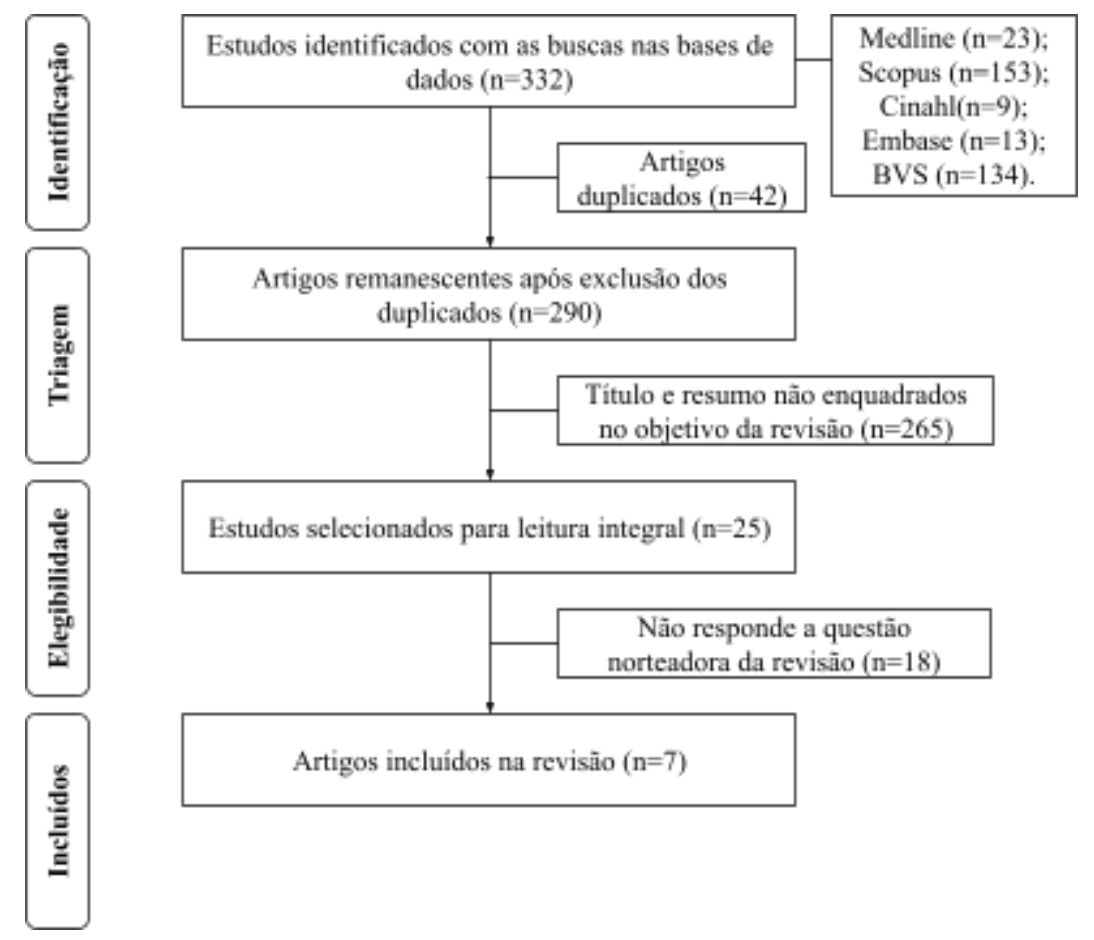

Posteriormente à seleção dos estudos incluídos, foi realizada a extração dos dados, que foram organizados em uma tabela. Nesta, foi exposto a identificação do artigo (autor, ano e país), as ações educativas encontradas, os principais resultados e o tipo de estudo. Ademais, os estudos foram categorizados pelo nível de evidência (NE) e grau de recomendação (GR), estabelecido pela Oxford Centre for Evidence-based Medicine (2009). Nisso, o NE é 


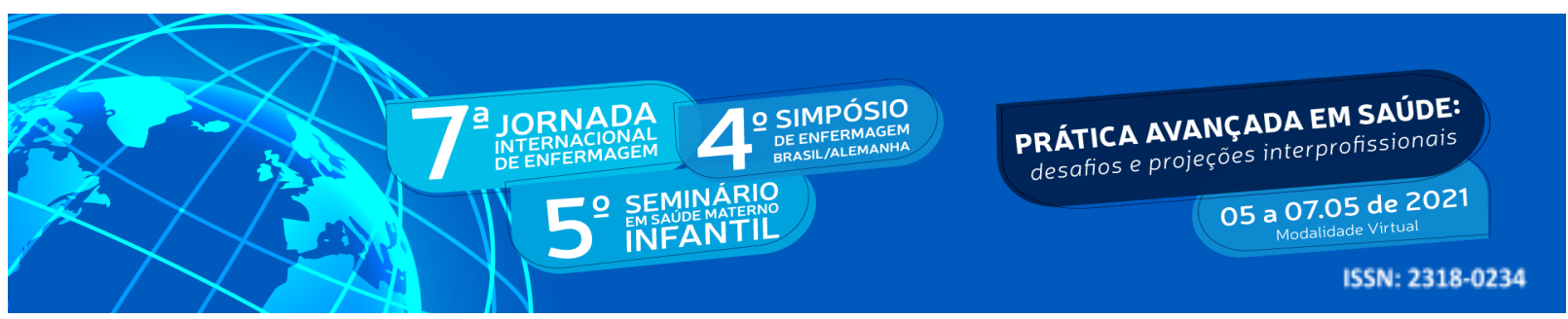

categorizado de 1 a 5, sendo o menor número referente ao melhor nível de evidência do artigo, e o GR variante de "A" a "D”, sendo "A" correspondente à melhor classificação.

\section{RESULTADOS E DISCUSSÃO}

A síntese dos resultados obtidos, a partir dos estudos incluídos na revisão, está exposta na Tabela 1. Nesta, observa-se informações referentes às ações educativas em saúde de cada estudo da amostra, bem como os principais resultados, considerando o desfecho da promoção da amamentação, a fim de evidenciar o impacto da primeira sobre a segunda. Ademais, nota-se que a maioria dos estudos da amostra são do Brasil (42,8\%), contemplando também artigos do Iraque (14,3\%), Irã (14,3\%), Estados Unidos da América (14,3\%) e Espanha (14,3\%); além disso, o ano de 2018 (57,1\%) destaca-se por ter tido maior número de publicações.

Tabela 1: Síntese dos estudos incluídos na amostra.

\begin{tabular}{c|c}
\hline $\begin{array}{c}\text { Autor / Ano / } \\
\text { País }\end{array}$ & Ações educativas em saúde \\
\hline
\end{tabular}

Piro; Ahmed

/ 2020 /

Iraque

Sessões educacionais sobre amamentação em grupo

Alves; Orientações sobre amamentação,

Oliveira; Rito baseado na Iniciativa Unidade

/ 2018 / Básica Amiga da Amamentação

Brasil (IUBAAM).

Intervenção educativa, com

Maia; Silva; elaboração de cartazes para

Moreira / exposições dialogadas, produção

2019 / Brasil de materiais representativos, vídeo do Ministério da Saúde.

\begin{tabular}{|c|c}
\hline Principais resultados & $\begin{array}{c}\text { Tipo de estudo } \\
/ \text { NE / GR }\end{array}$ \\
\hline
\end{tabular}

A autoeficácia da amamentação

no grupo experimental foi Estudo

significativamente maior, experimental/

apresentando maior nível de $3 \mathrm{~b}$ / B

conhecimento e atitude.

Associação positiva entre as Estudo

orientações e aleitamento

transversal / 4 /

materno exclusivo

$\mathrm{C}$

o conhecimento melhorou em

todos os blocos temáticos,

quando comparado ao

conhecimento prévio das

gestantes.

\section{Estudo}

longitudinal de intervenção / 4

/ C 


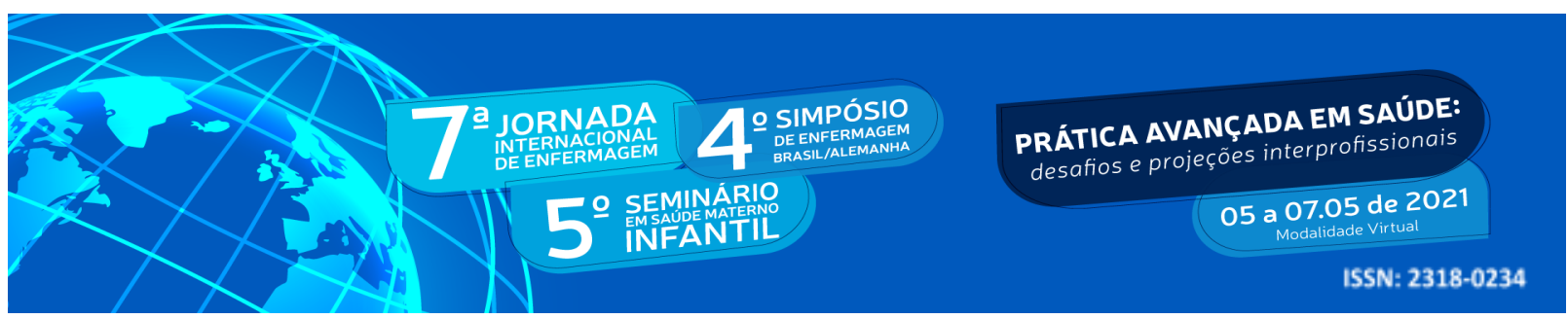

\begin{tabular}{|c|c|c|c|}
\hline $\begin{array}{l}\text { Araban et al. } \\
\text { / } 2018 \text { / Irã }\end{array}$ & $\begin{array}{l}\text { Intervenção baseada na } \\
\text { autoeficácia da amamentação, com } \\
\text { sessões de educação em grupo; } \\
\text { folheto informativo, imagens e } \\
\text { mensagens de texto. }\end{array}$ & $\begin{array}{l}\text { Maiores índices de AME e } \\
\text { autoeficácia na amamentação } \\
\text { entre os participantes do grupo } \\
\text { de intervenção. }\end{array}$ & $\begin{array}{l}\text { Ensaio clínico } \\
\text { controlado } \\
\text { randomizado / } \\
1 \mathrm{~b} / \mathrm{A}\end{array}$ \\
\hline $\begin{array}{l}\text { Harari et al. / } \\
2018 / \\
\text { Estados } \\
\text { Unidos da } \\
\text { América }\end{array}$ & $\begin{array}{l}\text { Educação sobre amamentação } \\
\text { baseada em evidências, por meio } \\
\text { de mensagens de texto } \\
\text { automatizadas. }\end{array}$ & $\begin{array}{l}\text { As mães do grupo de } \\
\text { intervenção tendem a ser mais } \\
\text { propensas a cumprir suas metas } \\
\text { de AME. }\end{array}$ & $\begin{array}{l}\text { Estudo piloto / } \\
2 \mathrm{~b} / \mathrm{B}\end{array}$ \\
\hline $\begin{array}{l}\text { Javorski et al. } \\
\text { / } 2018 \text { / } \\
\text { Brasil }\end{array}$ & $\begin{array}{l}\text { Educação por meio de álbum } \\
\text { seriado, utilizado para construir, } \\
\text { modelar e/ou alterar as crenças na } \\
\text { autoeficácia para amamentar. }\end{array}$ & $\begin{array}{l}\text { Efeito positivo na continuidade } \\
\text { do AME nas mulheres do grupo } \\
\text { intervenção em relação ao grupo } \\
\text { controle. }\end{array}$ & $\begin{array}{l}\text { Estudo de } \\
\text { intervenção, } \\
\text { controlado e } \\
\text { randomizado / } \\
2 \mathrm{~b} / \mathrm{B}\end{array}$ \\
\hline $\begin{array}{l}\text { Antoñanzas- } \\
\text { Baztán et al. / } \\
2020 \text { / } \\
\text { Espanha }\end{array}$ & $\begin{array}{l}\text { Promoção da autoeficácia da } \\
\text { amamentação, com informações, } \\
\text { vídeo, orientações específicas, } \\
\text { telefonema para aconselhamento } \\
\text { verbal. }\end{array}$ & $\begin{array}{l}\text { A manutenção da amamentação } \\
\text { foi estatisticamente significativa } \\
\text { no grupo de intervenção em } \\
\text { comparação com o grupo de } \\
\text { controle seis meses após o parto. }\end{array}$ & $\begin{array}{l}\text { Ensaio clínico } \\
\text { exploratório } \\
\text { multicêntrico } \\
\text { controlado / } \\
\text { 2b / B }\end{array}$ \\
\hline
\end{tabular}

A amamentação é um ato natural e aprendido, possível para a maioria das mães, desde que tenham acesso à informações adequadas, bem como um amparo atencioso do sistema de saúde, a fim de promover e proteger o aleitamento materno (MARTÍN-IGLESIAS et al., 2018). Dessa forma, colocar gestantes e puérperas em um papel de destaque propicia maiores direcionamentos sobre o processo educativo, possibilitando o compartilhamento de práticas e saberes em uma relação horizontal (GUERREIRO et al., 2014).

Ainda, sabe-se que a atenção primária à saúde configura-se como a base para capacitar os indivíduos para desenvolver a capacidade de decisão sobre sua saúde, por meio do compartilhamento de conhecimento, sabedoria e experiências. Nesse sentido, torna-se 


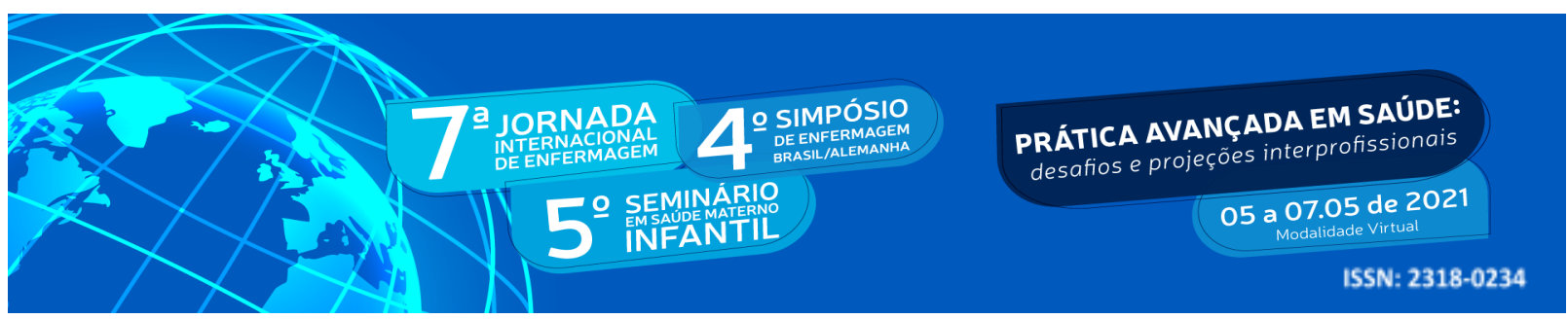

fundamental a realização de atividades educativas durante o parto, mas, principalmente, durante a gestação, uma vez que ao longo do pré-natal as mães devem ser orientadas a tomarem decisões para que possam vivenciar o parto de forma positiva, diminuindo os riscos de complicações no puerpério e sucesso no aleitamento materno (BARBOSA et al., 2015).

No entanto, as taxas de AME têm reduzido drasticamente em todo o mundo, especialmente, em países de baixa e média renda, mantendo-se abaixo das metas globais (KAVLE et al. 2017). No Brasil, pesquisas apontam que os índices de aleitamento materno exclusivo em menores de seis meses estagnaram durante os últimos anos, mantendo-se estável (36,6\%) desde 2013 (BOCCOLINI et al., 2017). Nesse contexto, evidencia-se que a prática da amamentação ainda se constitui como um grande desafio para o sistema de saúde, sobretudo, no tocante à implementação de ações intersetoriais de proteção e promoção do aleitamento materno e da adequada alimentação complementar (BRASIL, 2015).

Outrossim, estudos evidenciam que os principais fatores individuais relacionados à amamentação não exclusiva incluem fatores socioeconômicos, como baixa educação materna, idade materna mais jovem e diminuição da renda familiar; tal como os relacionados aos serviços de saúde, a exemplo, local do parto, frequência das consultas pré-natais, assistência ao parto, atraso no início da amamentação, entre outros (OGBO et al., 2018; WU et al., 2019).

Ademais, informações essenciais, relacionadas ao processo do aleitamento materno, são desconhecidas pelas mães, constituindo-se como um fator de risco, que necessita, portanto, de modificação (WU et al., 2019). Em um estudo realizado por Piro e Ahmed (2020), identificou-se que alguns pontos, associados ao conhecimento materno prévio, necessitam de intervenções educativas, tais como: benefícios do AM tanto para o bebê quanto para a mãe, benefícios do contato pele a pele, posição comum do AM, apego do bebê ao seio, sinais de sucção eficaz, métodos de ordenha de leite, entre outros.

Durante a assistência na APS, a equipe de saúde deve se apropriar da educação em saúde, ferramenta que objetiva melhorar a qualidade de vida do indivíduo ou do coletivo, por meio da articulação de saberes e práticas de promoção, prevenção e recuperação da saúde. Tal ferramenta surge como um impulsionador da autonomia do cuidado por orientar os usuários a tomarem atitudes favoráveis à saúde através da troca de saberes (TOSSIN et al., 2015). 


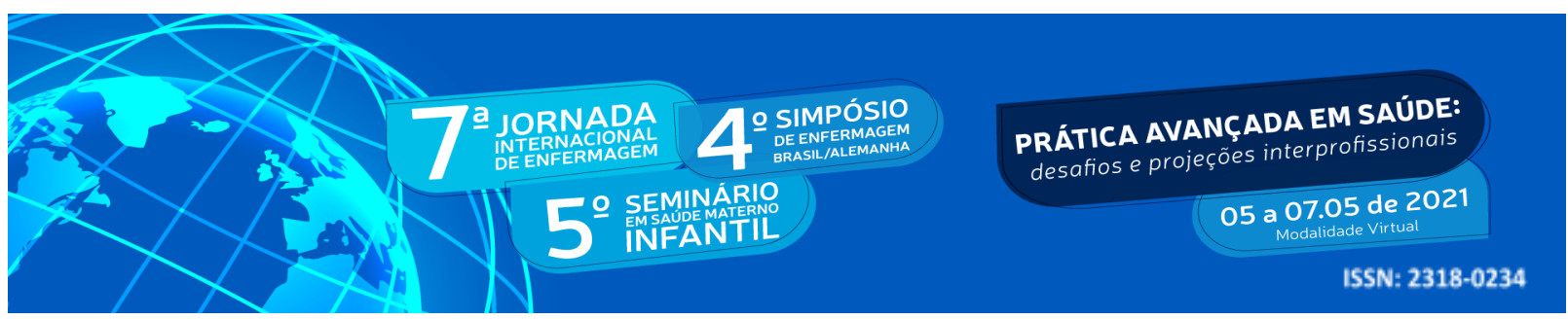

Diante disso, é indiscutível a necessidade de uma assistência pautada na educação em saúde, como estratégia para ensinar os casais que vivenciam o período gravídico-puerperal, obtendo posteriormente maiores taxas de sucesso na amamentação (ALVES et al., 2020). Corroborando com o exposto, pesquisas afirmam que a promoção e intensificação do AM previne cerca de 823.000 mortes de crianças a cada ano (VICTORIA et al. 2016).

Outrossim, identificou-se, nos artigos incluídos na presente revisão, o aumento nas taxas de amamentação após intervenções realizadas na APS com gestantes e/ou puérperas, evidenciando a importância e benefício das práticas de educação a essa clientela, propiciando a continuidade do AM, sobretudo, ao longo dos primeiros seis meses pós parto (ALVES; OLIVEIRA; RITO, 2018; ANTOÑANZAS-BAZTÁN et al., 2020). Em contrapartida, observou-se, em outros estudos das amostra, a ausência de análise do impacto das ações educativas até os 6 meses da criança - tempo de AME preconizado pela OMS - limitando a sua continuidade até os 2 meses (PIRO; AHMED, 2020; ARABAN et al., 2018; HARARI et al., 2018; JAVORSKI et al., 2018).

Por fim, ressalta-se as limitações desta pesquisa pela escassez de estudos que abordassem a educação em saúde para promoção da amamentação no contexto da APS, limitando a análise do assunto. Todavia, o presente estudo se mostra relevante por evidenciar a importância e fragilidades da educação em saúde para promover o incentivo ao aleitamento materno, gerando evidências para reflexão quanto à inferência das ações desenvolvidas.

\section{CONCLUSÕES}

O estudo mostrou que, durante os períodos de gestação e de puerpério, as mulheres necessitam adquirir conhecimento acerca do processo de aleitamento materno, para que possam garantir um desenvolvimento adequado à saúde da criança. Para tanto, as evidências científicas apontam a importância de uma assistência em saúde que ofereça, durante o processo de trabalho dos profissionais, sobretudo da APS, uma educação efetiva constante, de modo a promover, proteger e apoiar a amamentação pelas mães.

Diante disso, as intervenções educativas promovidas mostraram-se como ferramentas eficazes para a prática da amamentação. Nesse contexto, permitiu-se a inclusão das mães, bem como sua rede de apoio, no processo de aprendizagem, tornando-os protagonistas e produtores do conhecimento, de modo a promover a autoeficácia da amamentação. 


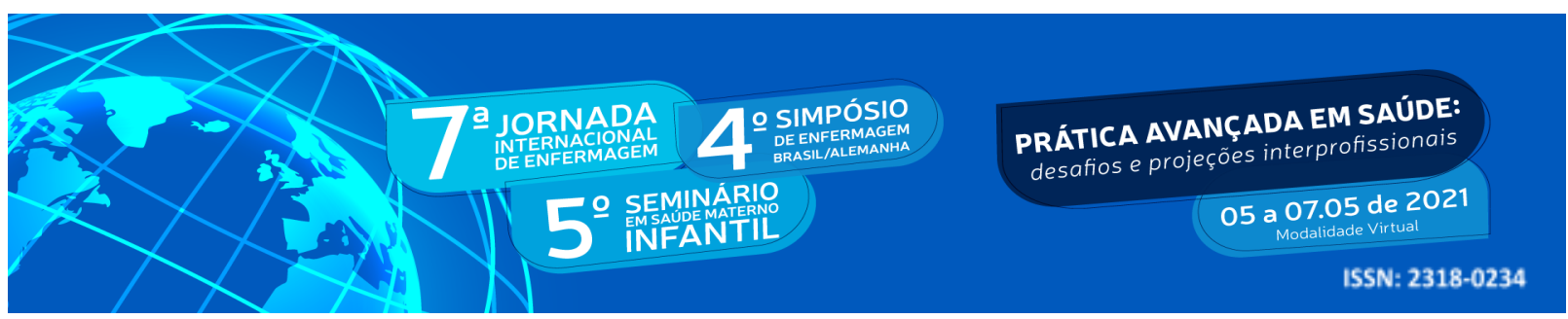

\section{REFERÊNCIAS}

ALVES, J. S; OLIVEIRA, M. I. C; RITO, R. V. V. F. Guidance on breastfeeding in primary health care and the association with exclusive breastfeeding. Ciência \& Saúde Coletiva, v. 23, n. 4, p. 1077-1088, 2018.

ALVES, Y. R. et al. A amamentação sob a égide de redes de apoio: uma estratégia facilitadora. Esc Anna Nery, v. 24, n. 1, p. e20190017, 2020.

ANTOÑANZAS-BAZT, E. et al. Six-month breastfeeding maintenance after a self-efficacy promoting programme: an exploratory trial. Scandinavian Journal of Caring Sciences, v. , n. , 2020.

ARABAN, M. et al. Randomized Controlled Trial of a Prenatal Breastfeeding Self-Efficacy Intervention in Primiparous Women in Iran. Journal of Obstetric, Gynecologic, \& Neonatal Nursing, v. 47, n. 2, p. 173-183, 2018.

BARBOSA, L. N. et al. Prevalence of educational practices about exclusive breastfeeding (EBF) in Cuiabá - MT. Escola Anna Nery, v. 19, n. 1, p. 147-153, 2015.

BOCCOLINI, C. S. et al. Tendência de indicadores do aleitamento materno no Brasil em três décadas. Rev Saúde Pública., v. 51, n. 108, p. 1-9, 2017.

BRASIL. Ministério da Saúde. Cadernos de Atenção Básica. Saúde da Criança:

Aleitamento Materno e Alimentação Complementar. 2ed. Brasília: Ministério da Saúde, 2015.

BRASIL. Ministério da Saúde. Gabinete do Ministro. Portaria no 1.920, de 5 de setembro de 2013. Brasília. 2013.

BRASIL. Ministério da Saúde. Rede Amamenta Brasil: Caderno do Tutor. Brasília: Ministério da Saúde, 2009.

GUERREIRO, E. M. et al. Educação em saúde no ciclo gravídico-puerperal: sentidos atribuídos por puérperas. Revista Brasileira de Enfermagem, v. 67, n. 1, p. 13-21, 2014.

HARARI, N. et al. Feasibility and acceptability of a text message intervention used as an adjunct tool by WIC breastfeeding peer counsellors: The LATCH pilot. Maternal Child Nutrition, v. 14, n. 1, p. 1-11, 2018.

JAVORSKI, M. et al. Efeitos de uma tecnologia educativa na autoeficácia para amamentar e na prática do aleitamento materno exclusivo. Revista da Escola de Enfermagem da USP, v. 52, p. e03329, p. 1-8, 2018. 


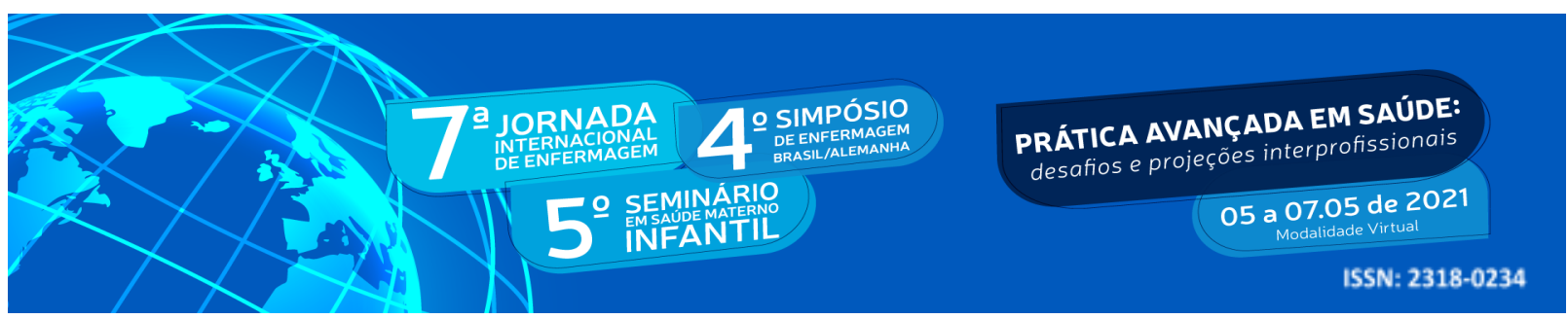

KAVLE, J. A. et al. Addressing barriers to exclusive breast-feeding in low- and middle-income countries: a systematic review and programmatic implications. Public Health Nutrition, v. 20, n. 17, p. 3120-3134, 2017.

MAIA, A. K; SILVA, B. Y. C; MOREIRA, L. C. J. Eficácia de intervenções educativas com gestantes sobre o grau de conhecimento em aleitamento materno. Brazilian journal in health promotion, v. 32, p. 1-9, 2019.

MARTÍN-IGLESIAS, S. et al. Effectiveness of an educational group intervention in primary healthcare for continued exclusive breast-feeding: PROLACT study. BMC Pregnancy and Childbirth, v. 18, n.1, p. 1-10, 2018.

MELO, L. C. O. et al. Primary health care attributes in breastfeeding care. Texto \& Contexto Enfermagem, v. 28, p. e20170516, 2019.

OGBO, F. A. et al. Population attributable risk of key modifiable risk factors associated with non-exclusive breastfeeding in Nigeria. BMC Public Health, v. 18, n. 1, p. 1-9, 2018.

ORGANIZAÇÃO MUNDIAL DA SAÚDE. Indicadores para evaluar las prácticas de alimentación del lactante y del niño pequeño. Suíça: Organização Mundial da Saúde, 2007.

PIRO, S. S; AHMED, H. M. Impacts of antenatal nursing interventions on mothers' breastfeeding self-efficacy: an experimental study. BMC Pregnancy and Childbirth, v. 20, n. 1, p. NA, 2020.

TASCA, R. et al. Recomendações para o fortalecimento da atenção primária à saúde no Brasil. Rev Panam Salud Publica, v. 44, p. 1-8, 2020.

TOSSIN, B. R. et al. Educational practices and self-care: evidence in scientific production of nursing. Revista Mineira de Enfermagem, v. 20, p. e940, 2015.

UNIVERSITY OF OXFORD. Oxford Center for Evidence-Based Medicine: Levels of Evidence. 2009. Disponível em:

$<$ https://www.cebm.ox.ac.uk/resources/levels-of-evidence/oxford-centre-for-evidence-basedmedicine-levels-of-evidence-march-2009>. Acesso em: 12 mar 2021.

VICTORIA, C. G. et al. Breastfeeding in the 21 st century: epidemiology, mechanisms, and lifelong effect. The Lancet, v. 387, p. 475-490, 2016.

VIEIRA, M. S. N; MATIAS, K. K; QUEIROZ, M. G. Educação em saúde na rede municipal de saúde: práticas de nutricionistas. Ciência \& Saúde Coletiva, v. 26, n. 2, p. 455-464, 2021.

WU, X. et al. Modifiable Individual Factors Associated with Breastfeeding: A Cohort Study in China. International Journal of Environmental Research and Public Health, v. 16, n. 5, p. 1-11, 2019. 